Physics

Physics Research Publications

\title{
Microcantilever mechanics in flowing viscous fluids
}
A. Jana
A. Raman
B. Dhayal

S. L. Tripp

R. G. Reifenberger

This paper is posted at Purdue e-Pubs.

http://docs.lib.purdue.edu/physics_articles/628 


\title{
Microcantilever mechanics in flowing viscous fluids
}

\author{
Anirban Jana and Arvind Raman ${ }^{\text {a) }}$ \\ School of Mechanical Engineering, Purdue University, West Lafayette, Indiana 47907 and Birck \\ Nanotechnology Center, Purdue University, West Lafayette, Indiana 47907 \\ Babita Dhayal, Steven L. Tripp, and Ronald G. Reifenberger \\ Department of Physics, Purdue University, West Lafayette, Indiana 47907 and Birck Nanotechnology \\ Center, Purdue University, West Lafayette, Indiana 47907
}

(Received 16 January 2007; accepted 9 February 2007; published online 16 March 2007)

\begin{abstract}
Microcantilevers are often deployed in flowing fluids to measure local flow velocities or to detect rapidly the nanomechanical binding of trace quantities of target analytes. The authors investigate the flow-induced mechanics of microcantilevers by deriving a semianalytical theoretical model for the nanoscale deflections of an elastic microcantilever due to a laminar viscous flow incident upon it. Conversely, the model allows for the estimation of the local flow velocities based on measured microcantilever deflection. Careful experiments performed on silicon microcantilevers in flowing nitrogen confirm the theoretical predictions up to a critical flow rate, beyond which unsteady flow-induced vibrations are seen to occur. (C) 2007 American Institute of Physics.
\end{abstract}

[DOI: $10.1063 / 1.2713238]$

Microcantilevers are versatile and highly sensitive sensors for nanoscale force, stress, and mass measurements. They are employed as the principal sensing element in scanning probe microscopy, ${ }^{1,2}$ the detection of chemical or biological analytes in gases or liquids (hydrogen, ${ }^{3}$ alcohol, ${ }^{3,4}$ DNA ${ }^{5,6}$ antibody, ${ }^{7}$ Bacillus subtilis spores ${ }^{8}$ ), calorimetry, ${ }^{4,9}$ humidity, ${ }^{4}$ and $p \mathrm{H}$ (Ref. 10) measurements, and flow sensing. ${ }^{11}$ In many of these applications, the sensing occurs via the nanomechanical bending of the microcantilever due to thermal or surface stresses.

Efforts to improve the sensitivity and throughput of microcantilever sensors in flowing fluids are primarily aimed toward fabricating more flexible microcantilevers and enabling higher flow rates. However, these tactics, in addition to decreasing the temperature stability, may also cause significant flow-induced bending and vibration of the highly flexible microcantilevers, and thus interfere with the analyte detection process. On the other hand, flow induced bending of microcantilevers may be exploited to develop ultrasensitive micromechanical flow sensors for high resolution, high bandwidth measurement of complex flow velocity fields. ${ }^{11,12}$ In this letter, we develop a semianalytical theoretical model for estimating viscous flow-induced deformations of microcantilevers. Our model may also be useful in understanding the hydrodynamic drag on a microcantilever moving through a fluid at a constant speed, an important consideration in single molecule force spectroscopy. ${ }^{13}$

The development of a theory of flow-induced cantilever bending was spurred by our experimental observations of significant bending and vibration of uncoated silicon microcantilevers placed in a stream of dry nitrogen gas (Fig. 1). The experiments measure the deflections of the individual microcantilevers at different flow rates. After an initial warm-up period, the flow rate is ramped from 20 to $150 \mathrm{~cm}^{3} / \mathrm{min}$ in steps of $10 \mathrm{~cm}^{3} / \mathrm{min}$. Short time histories of the deflection of the microcantilevers are captured for each flow rate. The average of each time history is taken to be the cantilever deflection at the corresponding flow rate. Tem-

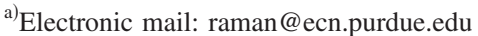

perature stability measurements verified that the observed flow-induced deflections are not due to thermal effects. ${ }^{14}$

Multiple data sets were collected for nominally identical arrays. The data sets exhibit good repeatability. Figure 1(c) shows a typical set of deflection data as a function of flow rate. The figure reveals a monotonic increase in cantilever deflection as the flow rate increases, with an onset of large amplitude oscillations beyond a critical flow rate that varied
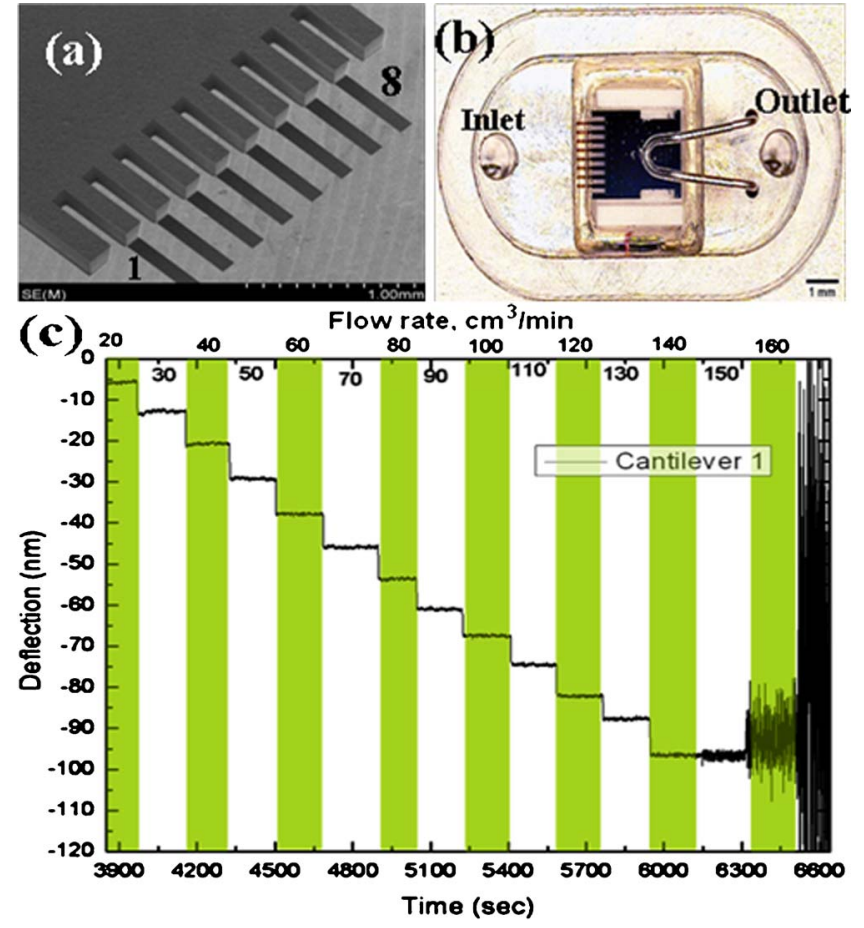

FIG. 1. (Color online) (a) Field emission scanning electron microscopy image showing an array of eight rectangular Si microcantilevers, each of length of $500 \mu \mathrm{m}$, width of $100 \mu \mathrm{m}$, thickness of $1 \mu \mathrm{m}$, and first natural frequency of approximately $5 \mathrm{kHz}$, used in the experiments. (b) Photograph of the flow cell (from Digital Instruments) with top cover removed. (c) Deflection of the cantilever No. 1 as a function of flow rate. Negative deflections correspond to upward deflections of the cantilever. The deflections are measured by an optical lever system. The flow rates are controlled by a flow controller at the flow cell inlet to within $0.5 \mathrm{~cm}^{3} / \mathrm{min}$ of set values. 


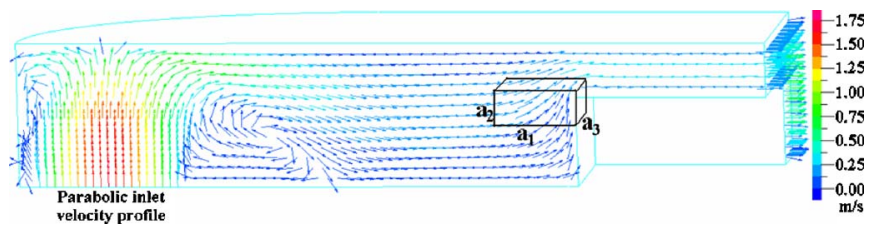

FIG. 2. (Color online) Three-dimensional simulation in ADINA of the flow inside the flow cell minus cantilevers. Only a front quarter of the flow cell is meshed with four-noded tetrahedral fluid elements. A parabolic velocity profile is prescribed at the flow cell inlet.

between 120 and $150 \mathrm{~cm}^{3} /$ min over the different data sets. Below this critical flow rate, a mere $10 \mathrm{~cm}^{3} /$ min change in flow rate increases the microcantilever deflection by 8 $10 \mathrm{~nm}$, a deflection comparable in magnitude to the deflection caused by surface stresses induced by analyte binding. Clearly it is difficult to make quantitative measurements of analyte induced surface stress in the presence of a flowing fluid. On the other hand, assuming a $1 \mathrm{~nm}$ optical lever resolution, the above microcantilever can measure flow velocities with high resolution $(\approx 3 \mathrm{~mm} / \mathrm{s})$.

It is difficult to construct a complete three-dimensional flow-structure interaction capable model for the microcantilever array in the flow cell. Instead, a three-dimensional computational fluid dynamics (CFD) model in ADINA (Ref. 16) is constructed to understand the flow inside an empty flow cell (Fig. 2). The boxed region in Fig. 2 indicates that the flow field in the immediate vicinity of the microcantilevers is approximately a uniform stream at an angle to the horizontal cantilevers.

This observation suggests a simpler model of a microcantilever placed in a uniform viscous stream at a nonzero angle of attack. Consider a cantilever of length $l$, width $b$, and thickness $h(l \gg b, l \gg h)$ placed in a free stream at an angle $\alpha$ [Fig. 3(a)]. The cantilever width is assumed normal to the free stream direction. Let $U_{\infty}$ be the free stream velocity, $f_{N}$ and $f_{L}$ be the normal and longitudinal hydrodynamic forces per unit length acting on the cantilever, $E$ be the cantilever Young's modulus, and $\rho$ and $v$ be the fluid density and kinematic viscosity. Since the cantilever deflection $w \ll l$, it can be assumed that $f_{N}$ remains constant over the entire length of the cantilever and the effect of $f_{L}$ on $w$ is negligible. Because $l \gg b$ and $l \gg h$, the changes in the flow field along the cantilever length can also be assumed negligible $(\partial / \partial z=0)$. With this assumption, the continuity and NavierStokes equations for an incompressible flow become

$$
\begin{aligned}
& u_{x, x}+u_{y, y}=0, \\
& u_{x, t}+u_{x} u_{x, x}+u_{y} u_{x, y}=-p_{, x}+v\left(u_{x, x x}+u_{x, y y}\right), \\
& u_{y, t}+u_{x} u_{y, x}+u_{y} u_{y, y}=-p_{, y}+v\left(u_{y, x x}+u_{y, y y}\right), \\
& u_{z, t}+u_{x} u_{z, x}+u_{y} u_{z, y}=v\left(u_{z, x x}+u_{z, y y}\right),
\end{aligned}
$$

where $\boldsymbol{u}=u_{x} \boldsymbol{i}+u_{y} \boldsymbol{j}+u_{z} \boldsymbol{k}$ and $p$ are the flow velocity and pressure fields. The notation $(\cdots)_{, x}$ represents $\partial(\cdots) / \partial x$ and so on. The key observation is that the first three equations, being decoupled from the fourth, completely determine $u_{x}, u_{y}$, and $p$, and hence $f_{N}$. In other words, $f_{N}$ decouples from the axial flow $^{15}$ and can be obtained from the purely twodimensional problem of a cantilever in a perpendicular cross flow. This two-dimensional problem is readily solved computationally in ADINA. ${ }^{16}$ The computational domain is shown Downloaded 03 Sep 2008 to 128.210.124.90. Redistribution subject
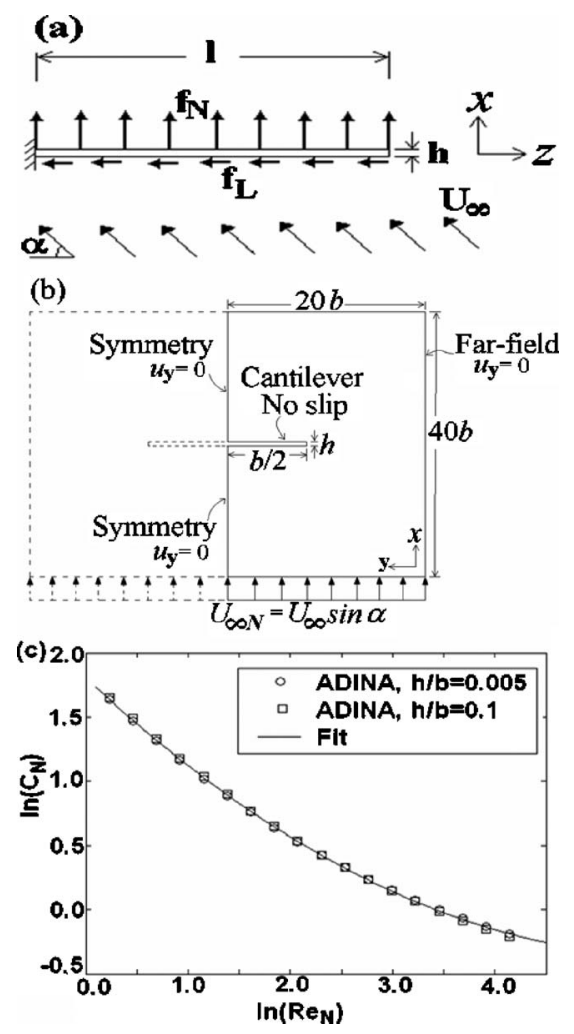

FIG. 3. (a) Schematic of the model for the cantilever in an oblique cross flow. (b) ADINA computational domain for analyzing the two-dimensional flow around a rigid cantilever placed in a perpendicular cross flow, to determine the coefficient of normal drag $C_{N}$ as a function of the Reynolds number $\operatorname{Re}_{N}$. The computational domain is meshed using three-noded triangular fluid elements. The values of $C_{N}$ extracted are independent of the overall size of the computational domain and the mesh fineness. (c) Variation of $C_{N}$ with $\mathrm{Re}_{N}$ for microcantilevers with two different cross-sectional aspect ratios. The solid line represents the fit to all the ADINA data for microcantilevers with 15 different aspect ratios in the range of 0.005 $\leqslant h / b \leqslant 0.1$.

schematically in Fig. 3(b). A comprehensive set of numerical simulations is performed in ADINA for a range of values of the Reynolds number $\mathrm{Re}_{N}=b U_{\infty N} / v$ and cantilever crosssectional aspect ratio $h / b\left(1 \leqslant \operatorname{Re}_{N} \leqslant 63\right.$ and $0.005 \leqslant h / b$ $\leqslant 0.1)$ typical for most current microcantilever sensor applications. For these parameter ranges, steady-state CFD analysis suffices.

The coefficient of normal drag, defined as $C_{N}$ $=f_{N} /\left(\rho U_{\infty N}^{2} b\right)$, measures the normal momentum transferred from the flow to the cantilever. Figure 3(c) shows the dependence of $C_{N}$ on $\mathrm{Re}_{N}$ for cross-sectional aspect ratios typical for slender microcantilevers $(0.005 \leqslant h / b \leqslant 0.1)$. Using linear regression, we find that a single quadratic relationship for the normal drag coefficient that is independent of the crosssectional aspect ratio,

$$
\ln \left(C_{N}\right)=\beta+\gamma \ln \left(\operatorname{Re}_{N}\right)+\delta\left(\ln \left(\operatorname{Re}_{N}\right)\right)^{2},
$$

with $\beta=1.805, \gamma=-0.7481$, and $\delta=0.06442$ provide an excellent fit to all the simulation data. ${ }^{17}$

The above method is easily extended to cantilevers with any uniform cross section, by accordingly modifying the computational domain. Equation (2) is expected to remain valid; however, the values of $\beta, \gamma$, and $\delta$ will change. For example, ADINA simulations show that for microcantilevers with circular cross sections, $\beta=2.794, \gamma=-0.8036$, and $\delta=0.05500$. This correlation is relevant for predicting the flow-induced deflections of artificial hair cells. ${ }^{12}$ The resultto AIP license or copyright; see http://apl.aip.org/apl/copyright.jsp 


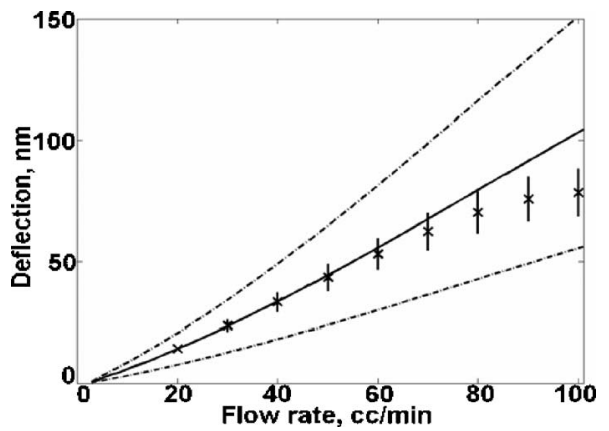

FIG. 4. Comparison between theoretically estimated and experimental measured deflections of the $500 \mu \mathrm{m}$ cantilevers. The solid curve represents the theoretical estimates. The dashed curves represent the error bounds for the theoretical estimates. For the theoretical calculations, $E_{\mathrm{Si}}=169 \mathrm{GPa}, \rho_{\mathrm{N} 2}$ $=1.616 \mathrm{~kg} \mathrm{~m}^{-3}, \nu_{\mathrm{N} 2}=1.081 \times 10^{-5} \mathrm{~m}^{2} / \mathrm{s}$. The crosses represent the mean microcantilever deflections from experiments. The vertical line segments represent \pm 1 standard deviation from the means. The base line for the experimental deflection data is that at $20 \mathrm{~cm}^{3} / \mathrm{min}$, which has been shifted to match with the theoretical estimate at $20 \mathrm{~cm}^{3} / \mathrm{min}$. 19 different sets of experimentally measured microcantilever deflections, representing several microcantilevers belonging to multiple array chips, have been employed to calculate the means and standard deviations.

ing dependence of $C_{N}$ on $\operatorname{Re}_{N}$ for the attack angle $\alpha=90^{\circ}$ compares excellently with published experimental data, ${ }^{15}$ providing further validation of our two-dimensional ADINA simulations.

The normal hydrodynamic force per unit length of the cantilever and Euler-Bernoulli formula for the tip deflection of a uniformly loaded cantilever are, respectively,

$$
\begin{aligned}
& f_{N}=\rho U_{\infty N}^{2} b C_{N}, \\
& w=\left(3 f_{N} l^{4}\right) /\left(2 E b h^{3}\right) .
\end{aligned}
$$

The flow-induced deflection of the microcantilever can be estimated by combining Eqs. (2)-(4). Alternatively, Eqs. (2) and (3) can be inverted to obtain the following closed form expression:

$$
U_{\infty N N}=\exp \left[-\left(\lambda+\sqrt{\lambda^{2}-4 \delta \mu}\right) /(2 \delta)\right],
$$

where $\quad \lambda=\gamma+2+2 \delta \ln (b / v) \quad$ and $\quad \mu=\beta+\gamma \ln (b / v)$ $+\delta(\ln (b / v))^{2}-\ln \left(f_{N} /(\rho b)\right)$ and $\beta, \gamma$, and $\delta$ are constants defined earlier. Given the microcantilever dimensions, Young's modulus, and the measured tip deflection, Eq. (4) can be inverted to determine $f_{N}$. This value can be used in Eq. (5) to estimate the flow normal velocity component $U_{\infty \infty N}$ in the vicinity of the microcantilever as is required in flow sensing.

Our theoretical model provides good estimates of the flow-induced deflections of the $500 \mu \mathrm{m}$ long cantilevers, as measured in Fig. 1(c). To obtain these estimates, the values of $U_{\infty}$ and $\alpha$ at different flow rates are required. These are obtained from the three-dimensional CFD simulation of the flow within the flow cell minus cantilevers (Fig. 2). At each flow rate, $U_{\infty}$ and $\alpha$ are estimated by averaging over the boxed flow region (with $a_{1}=550 \mu \mathrm{m}, a_{2}=50 \mu \mathrm{m}$, $\left.a_{3}=100 \mu \mathrm{m}\right)$. We find that $U_{\infty}$ increases linearly, whereas $\alpha$ is nearly constant $\left(\approx 39^{\circ}\right)$ for increasing flow rates in the range of $20-100 \mathrm{~cm}^{3} / \mathrm{min}$. Figure 4 compares the theoretically estimated cantilever bending with the experimental data, which are in good agreement for flow rates in the range of $20-100 \mathrm{~cm}^{3} / \mathrm{min}$. In fact, all our experimental data sets fall well within the upper and lower error bounds of the theoretical estimates. ${ }^{18}$
The proposed theory is inapplicable beyond a critical flow rate (high Reynolds numbers) when the flow around the microcantilever becomes unsteady, leading to microcantilever vibrations as observed in our experiments [Fig. 1(c)]. ${ }^{19}$ The vibration spectra show a large increase in amplitude of both the first and second bending modes of the microcantilevers at flow rates greater than the critical flow rate.

In summary, a theoretical model is introduced that predicts the hydrodynamic drag forces and bending of a microcantilever of uniform cross section due to a steady viscous flow incident upon it at an arbitrary angle of attack. The model also provides a formula [Eq. (5)] for estimating the local flow velocity around a microcantilever simply by measuring microcantilever bending. The work also has relevance to the prediction of flow-induced mechanics of artificial hair cells and hydrodynamic drag on microcantilevers in single molecule force spectroscopy.

This work received partial support from the NASA INAC Grant No. NCC2-1363; and the Sandia National Laboratories under Contract No. 623325, "Fluid-structure interactions in microsystems," through one of the authors (A.R.).

${ }^{1}$ G. Binnig and H. Rohrer, Helv. Phys. Acta 55, 726 (1982).

${ }^{2}$ G. Binnig, Ch. Gerber, and C. Quate, Phys. Rev. Lett. 56, 930 (1986).

${ }^{3}$ H. P. Lang, R. Berger, F. Battiston, J. P. Ramseyer, E. Meyer, C. Andreoli, J. Brugger, P. Vettiger, M. Despont, T. Mezzacasa, L. Scandella, H. J. Guntherodt, Ch. Gerber, and J. K. Gimzewski, Appl. Phys. A: Mater. Sci. Process. 66, S61 (1998).

${ }^{4}$ A. Boisen, J. Thaysen, H. Jensenius, and O. Hansen, Ultramicroscopy 82, 11 (2000).

${ }^{5}$ J. Fritz, M. K. Baller, H. P. Lang, H. Rothuzien, P. Vettiger, E. Meyer, H. J. Guntherodt, Ch. Gerber, and J. K. Gimzewski, Science 288, 316 (2000).

${ }^{6} \mathrm{G}$. Wu, H. Ji, K. Hansen, T. Thundat, R. Datar, R. Cote, M. F. Hagan, A. K. Chakraborty, and A. Majumdar, Proc. Natl. Acad. Sci. U.S.A. 98, 1560 (2001).

${ }^{7}$ R. Raiteri, G. Nelles, H. J. Butt, W. Knoll, and P. Skladal, Sens. Actuators B 61, 213 (1999).

${ }^{8}$ B. Dhayal, W. A. Henne, D. D. Doorneweerd, R. Reifenberger, and P. S. Low, J. Am. Chem. Soc. 128, 3716 (2006).

${ }^{9}$ J. K. Gimzewski, Ch. Gerber, E. Meyer, and R. R. Schlittler, Chem. Phys. Lett. 217, 589 (1994).

${ }^{10}$ H. F. Ji, K. M. Hansen, Z. Hu, and T. Thundat, Sens. Actuators B 72, 233 (2001).

${ }^{11}$ S. Barth, H. Koch, A. Kittel, J. Peinke, J. Burgold, and H. Wurmus, Rev. Sci. Instrum. 76, 075110 (2005).

${ }^{12}$ Y. Yang, N. Chen, C. Tucker, J. Engel, S. Pandya, and C. Liu, IEEE MEMS 2007, Kobe, Japan, 21-25 January 2007.

${ }^{13}$ H. Janovjak, J. Struckmeier, and D. J. Müller, Eur. Biophys. J. 34, 91 (2005).

${ }^{14}$ Temperature fluctuations at the flow cell outlet measured by a thermocouple were random, with a maximum variation of $\pm 0.2{ }^{\circ} \mathrm{C}$ over $2 \mathrm{~h}$. Also, comparing the deflections during flow rate ramp up and ramp down showed a drift of only $2-3 \mathrm{~nm}$.

${ }^{15}$ G. I. Taylor, Proc. R. Soc. London, Ser. A 214, 158 (1952).

${ }^{16}$ ADINA 8.2 Manuals, ADINA R\&D Inc. (www.adina.com), 2004.

${ }^{17}$ The parameters $\beta, \gamma$, and $\delta$ change by less than $5 \%$ over the range of $0.005 \leqslant h / b \leqslant 0.1$. For more accurate results, one may use the following phenomenological relationships extracted from our ADINA simulations: $\beta=1.797+0.2106(h / b), \quad \gamma=-0.7490+0.002412 \exp (-36.32(h / b))$, $\delta=0.06528-0.02207(h / b)$. Note that power law relationships between the drag coefficient and the Reynolds number are common in the literature. By setting $\delta=0$ in Eq. (2) and then proceeding with linear regression, a power law relationship can be obtained here as well. A poorer fit results.

${ }^{18}$ The following uncertainties are assumed in computing the error bounds: thickness $(h) 1 \%$, position of measurement $(x) 10 \%$, free stream velocity $\left(U_{\infty}\right) 5 \%$, attack angle $(\alpha) 5 \%$, and rest $0.1 \%$.

${ }^{19}$ Above the critical flow rate, the flow entering the flow cell remains laminar (Reynolds number $\approx 190$, based on the inlet radius of $0.625 \mathrm{~mm}$ ); and the cantilever natural frequencies remain far apart (no mode coalescence). 